\title{
A Universal Power-law Profile of Pseudo-Phase-Space Density-like Quantities in Elliptical Galaxies
}

\author{
Kyu-Hyun Chae \\ Department of Astronomy and Space Science, Sejong University, 98 Gunja-dong, \\ Gwangjin-Gu, Seoul 143-747, Republic of Korea \\ chae@sejong.ac.kr
}

\begin{abstract}
We study profiles of mass density, velocity dispersion (VD), and their combination using $\sim 2000$ nearly spherical and rotation-free SDSS galaxies. For observational stellar mass density $\rho_{\star}(r)$ we consider a range of dark matter $(\mathrm{DM})$ distribution $\rho_{\mathrm{DM}}(r)$ and VD anisotropy $\beta(r)$ to investigate radial stellar VD $\sigma_{\star \mathrm{r}}(r)$ using the spherical Jeans equation. While mass and VD profiles vary appreciably depending on DM distribution and anisotropy, the pseudo-phase-space densitylike combination $\rho(r) / \sigma_{\star r}^{3}(r)$ with total density $\rho(r)=\rho_{\star}(r)+\rho_{\mathrm{DM}}(r)$ is nearly universal. In the optical region the minus of its logarithmic slope has a mean value of $\langle\chi\rangle \approx 1.86-1.90$ with a galaxy-to-galaxy rms scatter of $\approx 0.04-0.06$, which is a few times smaller than that of $\rho(r)$ profiles. The scatter of $\chi$ can be increased by invoking wildly varying anisotropies that are, however, less likely because they would produce too large a scatter of line-of-sight VD profiles. As an independent check of this universality we analyze stellar orbit-based dynamical models of 15 ETGs of Coma cluster provided by J. Thomas. Coma ETGs, with $\sigma_{\star \mathrm{r}}(r)$ replaced by the rms velocity of stars $v_{\star \mathrm{rms}}(r)$ including net rotation, exhibit a similar universality with a slope of $\chi=1.93 \pm 0.06$. Remarkably, the inferred values of $\chi$ for ETGs match well the slope $\approx 1.9$ predicted by N-body simulations of DM halos. We argue that the inferred universal nature of $\rho(r) / \sigma_{\star \mathrm{r}}^{3}(r)$ cannot be fully explained by equilibrium alone, implying that some astrophysical factors conspire and/or it reflects a fundamental principle in collisionless formation processes.
\end{abstract}

Subject headings: galaxies: elliptical and lenticular, $\mathrm{cD}$ - galaxies: kinematics and dynamics - galaxies: structure 


\section{Introduction}

A useful approach in studies of galaxies is to look for any regularity or universality in the structure and then study astrophysical factors and/or principles responsible for it. Regarding galaxy profiles, until now much attention has been paid to possibility of a universal density profile of galaxies (e.g. Persic, Salucci \& Stel 1996; Gerhard et al. 2001; Loeb \& Peebles 2003; Koopmans et al. 2009; Remus et al. 2013). However, there is yet no reliable theoretical or numerical prediction on a universal mass profile of galaxies as existing models are flawed or incomplete and observational results on galactic mass profile are not converging well. On the observational side, there have been suggestions for approximately universal isothermal density profile $\rho(r) \propto r^{-2}$ for both spiral/disk galaxies (e.g. Rubin. Ford \& Thonnard 1980; Persic. Salucci \& Stel 1996) and early-type galaxies (ETGs; e.g. Gerhard et al. 2001; Humphrey \& Buote 2010; Koopmans et al. 2009). However, some recent results give a mean profile steeper-than-isothermal in ETGs (Chae, Bernardi \& Kravtsov 2013; Sonnenfeld et al. 2013; Bolton et al. 2012). Moreover, the density slope $\gamma \equiv-d \ln \rho(r) / d \ln r$ may vary with radius in both spirals (e.g. Persic. Salucci \& Stel 1996) and ellipticals (e.g. Chae, Bernardi \& Kravtsov 2013) or with redshift (Sonnenfeld et al. 2013; Bolton et al. 2012), and the intrinsic galaxyto-galaxy scatter is significant even within $R_{\mathrm{e}} 0.1<\sigma_{\gamma} \lesssim 0.2$ (e.g. Koopmans et al. 2009; Chae. Bernardi \& Kravtsov 2013). On the theoretical side, the classical isothermal model by Lvnden-Bell (1967) has several problems including infinite mass, indefinitely growing entropy (Lynden-Bell \& Wood 1968) and non-transitive nature (Arad \& Lynden-Bell 2005) and the applicability and usefulness of equilibrium statistical mechanics to gravitationally-bound collisionless systems are still being investigated (e.g. Tremaine. Henon \& Lvnden-Bell 1986; White \& Narayan 1987; He \& Kang 2010; Barnes \& Williams 2011; Pontzen \& Governato 2013). Hydrodynamic simulations of galaxy formation in the halo are used to study how the pristine (i.e. primitive before galaxy formation) halo is modified during the course of galaxy formation (e.g. Duffy et al. 2008; Gnedin et al. 2011; Macciò et al. 2012). However, physics involved in hydrodynamic simulations is so complex that a robust prediction on the mass profile is challenging. Therefore, the case for a universal density profile of galaxies as a dynamical attractor or fixed point in dynamical evolution (see, e.g., Loeb \& Peebles 2003) appears weak at present.

As first noticed by Taylor \& Navarro (2001), N-body simulations of hierarchical cold dark matter $(\mathrm{CDM})$ halo formation show that the combination $\rho_{\mathrm{pDM}}(r) / \sigma_{\mathrm{pDM}}^{3}(r)$ of mass density $\rho_{\mathrm{pDM}}(r)$ and velocity dispersion $(\mathrm{VD}) \sigma_{\mathrm{pDM}}(r)$ of the pristine DM halo, which is called pseudo phase-space (PPS) density because it has the dimension of the phase-space density (or distribution function) but is not a true measure of it (e.g., Ascasibar \& Binney 2005; Sharma \& Steinmetz 2005; Vass et al. 2009), is closer to universal than $\rho_{\mathrm{pDM}}(r)$ and can be well described by a scale-free power-law profile with slope $\chi_{\mathrm{pDM}} \equiv-d \ln \left[\rho_{\mathrm{pDM}}(r) / \sigma_{\mathrm{pDM}}^{3}(r)\right] / d \ln r \approx$ 
1.9 over three orders of magnitude in radius (e.g. Ascasibar et al. 2005; Austin et al. 2005; Wang \& White 2009; Vass et al. 2009; Navarro et al. 2010; Ludlow et al. 2011). It appears that $\rho_{\mathrm{pDM}}(r) / \sigma_{\mathrm{pDM}}^{3}(r)$ rather than $\rho_{\mathrm{pDM}}(r)$ offers a more powerful route to the universal nature of pristine halos. In this context, significant efforts have been made to investigate the physical origin of the universality, scale-free nature and slope value of the $\rho_{\mathrm{pDM}}(r) / \sigma_{\mathrm{pDM}}^{3}(r)-$ profile (e.g. Tavlor \& Navarro 2001; Austin et al. 2005; Barnes et al. 2006; Wang \& White 2009; Vass et al. 2009; Ludlow et al. 2011; Lapi \& Cavaliere 2011) and their implication for the structure of pristine DM halos (e.g. Austin et al. 2005; Dehnen \& McLaughlin 2005).

In this work we examine mass and VD profiles of galaxies and their combination using SDSS elliptical galaxies. It is argued that there exists a universal profile of PPS density-like quantities in elliptical galaxies, akin to the universal PPS density profile of pristine DM halos, with an intrinsic scatter smaller than the observed scatter of mass density profiles.

\section{Samples of Early-type Galaxies and Their Models}

We consider a sample of nearly spherical galaxies that allows a relatively straightforward analysis. The galaxy sample is drawn from the Sloan Digital Sky Survey (SDSS: Chae, Bernardi \& Kravtsov (2013) and references theirin) and contains $\sim 2000$ nearly spherical (surface brightness ellipticity $<0.15$ ) and disk-less (disk mass is within the measurement error of the bulge mass) galaxies at redshifts $z \lesssim 0.25$. Each galaxy is assumed to be in a dynamical equilibrium state satisfying the spherical Jeans equation (Binney \& Tremaine 2008) given by

$$
\frac{d\left[\rho_{\star}(r) \sigma_{\star \mathrm{r}}^{2}(r)\right]}{d r}+2 \frac{\beta(r)}{r}\left[\rho_{\star}(r) \sigma_{\star \mathrm{r}}^{2}(r)\right]=-G \frac{\rho_{\star}(r) M(r)}{r^{2}},
$$

where $\rho_{\star}(r)$ is the volume stellar mass distribution, $M(r)$ is the total mass within $r, \sigma_{\star r}(r)$ is the radial stellar $\mathrm{VD}$, and $\beta(r)$ is the $\mathrm{VD}$ anisotropy given by $\beta(r)=1-\left[\sigma_{\star \theta}^{2}(r)+\sigma_{\star \phi}^{2}(r)\right] /\left[2 \sigma_{\star \mathrm{r}}^{2}(r)\right]$ where $\sigma_{\star \theta}(r)$ and $\sigma_{\star \phi}(r)$ are tangential VDs of stars in spherical coordinates. The Jeans equation can be used to obtain a VD profile $\sigma_{\star \mathrm{r}}(r)$ for an observationally inferred $\rho_{\star}(r)$ if $\rho_{\mathrm{DM}}(r)$ and $\beta(r)$ are known or specified. Conversely, if a line-of-sight (LOS) VD profile $\sigma_{\star \text { klos }}(r)$ is measured for some radial range, the Jeans equation can be used to derive $\rho_{\mathrm{DM}}(r)$ and $\beta(r)$ along with $\sigma_{\star \mathrm{r}}(r)$ for the observed radial range. Without $\sigma_{\star l \mathrm{los}}(r)$ for SDSS galaxies, we take the former approach and consider a range of $\rho_{\mathrm{DM}}(r)$ and $\beta(r)$. For DM distribution we consider both the case of no DM and assigning a generalized Navarro-Frenk-White (gNFW) halo $\rho_{\mathrm{gNFW}}(r) \propto r^{-\alpha}\left(1+r / r_{\mathrm{s}}\right)^{-3+\alpha}$ using a wealth of empirical information, as

in Chae. Bernardi \& Kravtsov (2013), including statistical distribution of LOSVD profiles within $\sim R_{\mathrm{e}}$ from well-studied ETGs and statistical properties and relations of ETGs and DM halos. We consider both constant anisotropy and radially varying anisotropy using a 
function of the form $\beta(r)=\beta_{1} /\left(1+r_{1}^{2} / r^{2}\right)+\beta_{2} /\left(1+r_{2}^{2} / r^{2}\right)$, which is a combination of two Osipkov-Merritt-type (Binney \& Tremaine 2008) models but allows an extremum at a finite radius. We take anisotropy values randomly from an observed distribution described in section 2.7 of Chae, Bernardi \& Kravtsov (2013). For the case of radially varying anisotropy the mean anisotropy for $r<R_{\mathrm{e}}$ and the anisotropy at infinity take a common value drawn from the observed distribution and $0<r_{1}<0.5 R_{\mathrm{e}}$ and $r_{1}<r_{2}<R_{\mathrm{e}}$ are assigned so that an extremum occurs within $R_{\mathrm{e}}$. Radially varying anisotropies with an extremum (or extrema) within $R_{\mathrm{e}}$ result often from dynamical modeling of ETGs (e.g. Gerhard et al. 2001; Thomas et al. 2007).

We also consider a small number of ETGs of the Coma cluster for which $\sigma_{\text {los }}(r)$ has been individually measured up to $>R_{\mathrm{e}}$ and thus $\rho_{\mathrm{DM}}(r)$ and $\beta(r)$ along with $\sigma_{\star \mathrm{r}}(r)$ have been derived from the data (Thomas et al. 2007, 2009b). Specifically, two-component axisymmetric galaxy models are used and a best-fit model is calculated through a maximum entropy implementation of Schwarzschild's stellar orbit superposition technique fitting the observed LOSVD profile. The detailed modeling results are provided by J. Thomas (private communication). The Coma sample consists of 15 ETGs selected from 19 ETGs (Thomas et al. 2007, $2009 \mathrm{~b})$. Our criterion for this selection is that the mean of the radial ranges along the major and minor axes over which LOSVD profiles were observed extends beyond $R_{\mathrm{e}} / 2$. Seven of them (GMP 144, 282, 1750, 3510, 3792, 5279 and 5975) are ellipticals without significant disks. The rest are two ellipticals (GMP 2440, 3958) possessing significant disks and six lenticulars (GMP 756, 1176, 1990, 2417, 3414, and 4928). The excluded galaxies are GMP 2921, 3329, 4822 and 5568, three of which are cD/D galaxies. Because streaming motion can be significant for lenticulars and ellipticals with non-negligible disks, we consider an average 1-dimensional second velocity moment given by

$$
v_{\star \mathrm{rms}}(r)=\left[\frac{\sigma_{\star \mathrm{r}}^{2}(r)+\sigma_{\star \theta}^{2}(r)+\sigma_{\star \phi}^{2}(r)+\bar{v}_{\star \phi}^{2}(r)}{3}\right]^{1 / 2}=\left[\sigma_{\star}^{2}(r)+\frac{1}{3} \bar{v}_{\star \phi}^{2}(r)\right]^{1 / 2},
$$

where $\bar{v}_{\star \phi}(r)$ is the net intrinsic rotation speed of stars at $r$ taking into account the inclination of each Coma galaxy (Thomas et al. 2007; J. Thomas, private communication). Notice that $v_{\star \mathrm{rms}}(r)=\sigma_{\star}(r)$ if $\bar{v}_{\star \phi}(r)=0$.

\section{Profiles of Density $\rho(r)$, Velocity Dispersion $\sigma_{\star \mathrm{r}}(r)$ and $\rho(r) / \sigma_{\star \mathrm{r}}^{3}(r)$}

If a quantity $\rho(r) / \sigma_{\star \mathrm{r}}^{\epsilon}(r)$ should follow a universal power-law profile at least for some radial range, then we expect a good correlation between slopes $\gamma \equiv-d \ln \rho(r) / d \ln r$ and $\eta \equiv$ $-d \ln \sigma_{\star \mathrm{r}}(r) / d \ln r$ for some radial range. Here the exponent $\epsilon$ is unknown but a theoretically interesting value is $\epsilon=3$ for which the quantity has the dimension of phase space density. 
If we write $\rho(r) / \sigma_{\star \mathrm{r}}^{\epsilon}(r) \propto r^{-a}$, then we expect $\gamma=a+\epsilon \eta$ for some radial range. To test this hypothesis we calculate values of $\gamma$ and $\eta$ and check if there is a correlation. For the SDSS sample we consider a fixed radial range of $0.1 R_{\mathrm{e}}<r<R_{\mathrm{e}}$ as this range is most relevant for the modeling results (Chae, Bernardi \& Kravtsov 2013). For the Coma cluster sample we use the mean of the radial ranges of observed LOSVD profiles along the major and minor axes, which varies from galaxy to galaxy but is typically $0.1 R_{\mathrm{e}} \lesssim r \lesssim 1.5 R_{\mathrm{e}}$. For Coma galaxies having net rotations $\sigma_{\star \mathrm{r}}(r)$ is replaced by $v_{\star \mathrm{rms}}(r)$ (Equation 2).

The left panel of Figure1 displays the correlation between $\gamma$ and $\eta$. Good correlations are found independently from both samples. The least-square fit relations are $\gamma \approx 1.87+2.83 \eta$ (constant anisotropy), $\gamma \approx 1.91+2.63 \eta$ (varying anisotropy) for the SDSS sample and $\gamma \approx 1.90+3.29 \eta$ for the Coma sample. Similar results are found even for the case of no DM [i.e. $\left.\rho(r)=\rho_{\star}(r)\right]$ in which stars are self-gravitating. These results imply that a PPS densitylike combination with $\epsilon=3$ is expected to be (close to) universal with a power-law exponent of $\approx-1.9$, which interestingly matches well the slope -1.875 predicted by the classical selfsimilar spherical-infall model (Bertschinger 1985; Fillmore \& Goldreich 1984). The middle and right panels of Figure 1 display the distributions of $\chi \equiv-d \ln \left[\rho(r) / \sigma_{\star \mathrm{r}}^{3}(r)\right] / d \ln r$ against $\gamma$ and $\eta$. The slope $\chi$ is not correlated with either $\gamma$ or $\eta$. This lack of correlation of $\chi$ is what would be expected if $\rho(r) / \sigma_{\star \mathrm{r}}^{3}(r)$ should be universal.

The large number of galaxies in the SDSS sample allows us to investigate how slopes are distributed as a function of various parameters. Figure 2 displays the distributions of $\gamma$, $\eta$ and $\chi$ against stellar mass $M_{\star}$, Sérsic (Sérsic 1968) index $n$, effective radius $R_{\mathrm{e}}$, projected stellar mass density $\Sigma_{\mathrm{e}} \equiv\left(M_{\star} / 2\right) /\left(2 \pi R_{\mathrm{e}}^{2}\right)$ within $R_{\mathrm{e}}$ and the host halo virial mass $M_{200}$. Only the results with constant anisotropies are displayed here but the case of varying anisotropy is qualitatively similar. It is evident that $\chi$ is little correlated with any galactic or halo parameter. In contrast $\gamma$ varies systematically in particular with $R_{\mathrm{e}}$ and $\Sigma_{\mathrm{e}}$. Slope $\eta$ also exhibits some systematic trends but to a lesser degree. The mean and rms scatter of the slopes are: $\langle\gamma\rangle=2.13, s_{\gamma}=0.13 ;\langle\eta\rangle=0.09, s_{\eta}=0.05 ;$ and, $\langle\chi\rangle=1.86, s_{\chi}=0.04$. Remarkably, slope $\chi$ has much smaller scatter compared with $\gamma$ as can be clearly seen in the histogram (top left panel) of Figure 2, The scatter of $\chi$ increases up to 0.06-0.08 for general ETGs of any ellipticity for constant anisotropies and further increases by $0.01-0.02$ for the adopted radially varying anisotropy. The scatter of $\chi$ can be increased even up to or beyond the scatter of $\gamma$ by considering wildly varying anisotropies which however predict too large scatters of LOSVD profiles. The small scatter of $\chi$ is also suggested by the Coma cluster sample for which we have: $\langle\gamma\rangle=2.13, s_{\gamma}=0.14 ;\langle\eta\rangle=0.07, s_{\eta}=0.04 ;$ and, $\langle\chi\rangle=1.93$, $s_{\chi}=0.06$. Therefore, it appears that $\rho(r) / \sigma_{\star \mathrm{r}}^{3}(r)$ (or $\left.\rho(r) / v_{\star \mathrm{rms}}^{3}(r)\right)$ is closer to universal than $\rho(r)$ at least within $\sim R_{\mathrm{e}}$. 
Figure 3 displays the predicted profiles up to $10 R_{\mathrm{e}}$ based on the SDSS sample. Compared with $\rho(r) \rho(r) / \sigma_{\star \mathrm{r}}^{3}(r)$ is much closer to a universal power-law profile. Figure 4 displays the profiles of 15 Coma cluster ETGs directly constrained by the individually measured LOSVDs. These profiles independently indicate that for ellipticals $\rho(r) / \sigma_{\star \mathrm{r}}^{3}(r)$ is closer to universal than $\rho(r)$. What is even more striking from the Coma profiles is that all ETGs including lenticulars appear to follow the universal profile when $v_{\star \mathrm{rms}}(r)$ (Equation 2) rather than $\sigma_{\star \mathrm{r}}(r)$ is used.

Unlike $\rho(r) / \sigma_{\star \mathrm{r}}^{3}(r)$, the quantity $\rho_{\star}(r) / \sigma_{\star \mathrm{r}}^{3}(r)$ does not exhibit a universality for a realistic galaxy embedded in a DM halo. The scatter of the slope $-d \ln \left[\rho_{\star}(r) / \sigma_{\star \mathrm{r}}^{3}(r)\right] / d \ln r$ is even larger than that of $\gamma$. This property is rooted in the fact that stellar motions (DM motions as well) are not self-gravitating but governed by the total mass distribution, as can be seen in Equation (1). In a pristine DM halo in which DM motions are governed by its own gravity the PPS density $\rho_{\mathrm{pDM}}(r) / \sigma_{\mathrm{pDM}}^{3}(r)$ exhibits a universality (e.g. Taylor \& Navarro 2001; Austin et al. 2005; Ludlow et al. 2011; Navarro et al. 2010). However, in 'real' DM halos hosting galaxies the PPS density of DM particles $\rho_{\mathrm{DM}}(r) / \sigma_{\mathrm{DM}}^{3}(r)$ is not universal or power-law as can be shown using our models. This property was also noticed in cosmological hydrodynamic simulations (e.g. Zemp et al. 2012).

\section{A Simple Analysis of Equilibrium}

Let us analyze the spherical Jeans equation to see if the universality of $\rho(r) / \sigma_{\star r}^{3}(r)$ can be a natural outcome of equilibrium. Using Equation (1) one can express a PPS density-like quantity with the radial VD as follows:

$$
\frac{y(x)}{z^{3}(x)}=\frac{1}{\kappa} \frac{\gamma_{\star}(x)+2 \eta(x)-2 \beta(x)}{x^{2} z(x)}\left(1-2 \eta(x)+\frac{d \ln \left[\gamma_{\star}(x)+2 \eta(x)-2 \beta(x)\right]}{d \ln x}\right),
$$

where $x \equiv r / r_{0}, y(x) \equiv \rho(r) / \rho\left(r_{0}\right), z(x) \equiv \sigma_{\star \mathrm{r}}(r) / \sigma_{\star \mathrm{r}}\left(r_{0}\right)$, and $\kappa \equiv 4 \pi G \rho\left(r_{0}\right) r_{0}^{2} / \sigma_{\star \mathrm{r}}^{2}\left(r_{0}\right)$ with an arbitrary reference radius $r_{0}$ (see Taylor \& Navarro (2001)). In Equation (3) the following symbols are used: $\gamma_{\star}(x) \equiv-d \ln y_{\star}(x) / d \ln x$ with $y_{\star}(x) \equiv \rho_{\star}(r) / \rho_{\star}\left(r_{0}\right)$, and $\eta(x) \equiv$ $-d \ln z(x) / d \ln x$.

The last factor in the large parenthesis of the right-hand side of Equation (3) is $\approx 1$. In the numerator of the second factor of the right-hand side $\gamma_{\star}\left(\approx 0.9+1.9\left(r / R_{\mathrm{e}}\right)^{1 / n} \gtrsim 2\right.$ for $r>0.1 R_{\mathrm{e}}$ assuming the Sérsic profile) is dominating as $|\eta| \lesssim 0.2$ and $|\beta| \lesssim 0.5$ in most cases. For some radial range, e.g. $0.1 R_{\mathrm{e}}<r<R_{\mathrm{e}}$, we may write $z(x)=x^{-\eta}$ to a good approximation. We may also write the universal PPS density-like profile as $y(x) / z^{3}(x)=$ $x^{-\chi}$. Then we are left with $\chi \approx 2-\eta$. Similarly, if we write $y(x)=x^{-\gamma}$ for $0.1 R_{\mathrm{e}}<r<R_{\mathrm{e}}$, we obtain $\chi \approx 3-0.5 \gamma$. These relations can be used to predict a mean value $\langle\chi\rangle \approx 1.9$ 
by empirical values of $\langle\eta\rangle \approx 0.1$ or $\langle\gamma\rangle \approx 2.1$. They also imply that the intrinsic scatter of $\chi$ is comparable to that of $\eta$ but one half of that of $\gamma$. These predictions of the mean and the intrinsic scatter are consistent with the results from our galaxy models. However, as shown in the middle and right panels of Figure 1 these approximate relations are not obeyed by our galaxy models. Our galaxy models show no correlation of $\chi$ with either $\eta$ or $\gamma$. Therefore, although equilibrium can predict the mean and the scatter of the slope of $\rho(r) / \sigma_{\star \mathrm{r}}^{3}(r)$, equilibrium itself cannot generically explain its universal nature seen in our models. For simple galaxy models equilibrium demands correlations of $\chi$ with $\gamma$ and $\eta$. The seen universality free of such correlations implies that some other astrophysical factors and/or principles are at work in (our models of) real galaxies.

For a galaxy with net rotation one may assume that stars in a local volume centered at $r$ satisfy a virial equation $v_{\star \mathrm{rms}}^{2}(r)=-\Phi(r)$ (similar to the equation for the whole galaxy) where $v_{\star \mathrm{rms}}(r)$ is given by Equation (2) and $\Phi(r)$ is the gravitational potential. From this equation similar relations of $\chi$ with $\eta$ and $\gamma$ can be obtained.

\section{Discussion}

A range of DM distribution and VD anisotropy in elliptical galaxies have been considered for investigating galaxy mass and VD profiles. The PPS density-like combination $\rho(r) / \sigma_{\star \mathrm{r}}^{3}(r)$

(or $\rho(r) / v_{\star \mathrm{rms}}^{3}(r)$ for general ETGs) appears to be closer to universal than $\rho(r)$. Moreover, the universality of $\rho(r) / \sigma_{\star \mathrm{r}}^{3}(r)$ of ellipticals closely parallels the universality of the PPS density of pristine DM halos (e.g. Austin et al. 2005; Navarro et al. 2010; Ludlow et al. 2011) with the values of slope $\chi$ agreeing well. The PPS density is known to be scale-free over three orders of magnitude in radius for N-body simulated halos. Our galaxy models indicate a power-law profile of $\rho(r) / \sigma_{\star \mathrm{r}}^{3}(r)$ in the optical region, and extrapolation from our models suggests a scale-free profile over two orders of magnitude in radius. It appears then natural to suggest that the PPS density-like quantity $\rho(r) / \sigma_{x \mathrm{r}}^{3}(r)$ for any collisionless component $x$ in dynamical equilibrium follows a universal power-law profile at least for some radial range.

Examination of Jeans (or virial) equation shows that equilibrium can predict $\chi=3-$ $0.5 \gamma$ giving a mean slope of $\langle\chi\rangle \approx 1.9$ for an empirical $\langle\gamma\rangle \approx 2.1$ in the optical region. However, equilibrium cannot generically predict a power-law profile or the lack of correlation of $\chi$ with galactic properties. What astrophysical factors and/or physical principles can be attributed to the shared universality of $\rho(r) / \sigma_{x \mathrm{r}}^{3}(r)$ in N-body simulated pristine halos and our ellipticals? Total mass profiles have no similarity between them. Moreover, the physical formation process is quite different. The pristine halo has formed through fast collapse, major and minor mergers, and accretion. Only dissipationless processes are involved. On 
the other hand, an ETG is believed to form through merging of disk galaxies and/or preexisting ETGs. In the formation of the stellar disk dissipational physics plays the essential role and consequently the total density deviates from the NFW (Navarro, Frenk \& White 1997) profile of the pristine halo and gets much steeper in the central region. The shared universality of $\rho(r) / \sigma_{x \mathrm{r}}^{3}(r)$ despite large differences in mass profiles, structures and formation histories between pristine DM halos and ETGs suggests that the universality is not an outcome of specific astrophysical factors but reflects a fundamental physical principle/nature governing collisionless systems. For Coma cluster ETGs maximum entropy models ignoring observed LOSVD profiles, as shown in the right-most panel of Figure 4, resemble best-fit

models in a statistical sense. This result is in line with the finding by Thomas et al. (2009a) that flattening of an ETG by stellar anisotropy maximizes the entropy for a given density distribution. However, the relevance of maximum entropy principle for collisionless systems under gravity is not well understood at present. Perhaps, dynamical mixing/relaxation may be responsible for the universality (see, e.g., Valluri et al. 2007).

While $\rho(r) / \sigma_{x \mathrm{r}}^{3}(r)$ appears to be universal, total mass density $\rho(r)$ (not to mention the component density $\rho_{x}(r)$ ) is determined by other physics. For example, purely radial collapse scenario by Bertschinger (1985) gives $\rho(r) \propto r^{-2.25}$ while inclusion of non-radial motions can give a profile similar to the NFW (e.g. Ascasibar et al. 2005; MacMillan, Widrow \& Henriksen 2006; Vogelsberger, Mohavaee \& White 2011). In ETGs baryonic physics is responsible for the steep profile $\rho(r) \propto r^{-\gamma}$ with $\gamma \sim 2.1$ in the optical region.

The author is indebted to Jens Thomas for providing him with unpublished results on Coma cluster ETGs and useful discussions. The author thanks Andrey Kravtsov for useful discussions and helpful comments on the manuscript. The author also would like to thank the anonymous referees for useful comments.

\section{REFERENCES}

Arad, I., \& Lynden-Bell, D. 2005, MNRAS, 361, 385

Ascasibar, Y. \& Binney, J. 2005, MNRAS, 356, 872

Ascasibar, Y., Yepes, G., Gottlöber, S., \& Müller, V. 2004, MNRAS, 352, 1109

Austin, C. G., Williams, L. L. R., Barnes, E. I., Babul, A., \& Dalcanton, J. J. 2005, ApJ, 634,756

Barnes, E. I., Williams, L. L. R., Babul, A., \& Dalcanton, J. J. 2006, ApJ, 643, 797 
Barnes, E. I., \& Williams, L. L. R. 2011, ApJ, 728, 136

Bertschinger, E. 1985, ApJS, 58, 39

Binney, J. \& Tremaine S. 2008, Galactic Dynamics, 2nd ed. (Princeton Univ. Press, Princeton, NJ).

Bolton, A. S. et al., 2012, ApJ, 757, 82

Chae, K. H., Bernardi, M., \& Kravtsov, A. V. 2014, MNRAS, 437, 3670

Dehnen, W. \& McLaughlin, D. E., 2005, MNRAS, 363, 1057

Duffy, A. R., Schaye, J., Kay, S. T., \& Dalla Vecchia, C. 2008, MNRAS, 390, 64

Fillmore, J. A., \& Goldreich, P. 1984, ApJ, 281, 1

Gerhard, O., Kronawitter, A., Saglia, R. P., \& Bender, R. 2001, AJ, 121, 1936

Gnedin, O. Y., Ceverino, D., Gnedin, N. Y., Klypin, A. A., Kravtsov, A. V., Levine, R., Nagai, D., \& Yepes, G. 2011, ApJ, submitted (arXiv:1108.5736)

He, P., \& Kang, D.-B. 2010, MNRAS, 406, 2678

Humphrey, P. J. \& Buote, D. A. 2010, MNRAS, 403, 2143

Huss, A., Jain, B., \& Steinmetz, M. 1999, ApJ, 517, 64

Koopmans, L. V. E., et al. 2009, ApJ, 703L, 51

Lapi, A., \& Cavaliere, A. 2011, ApJ, 743, 127

Loeb, A., \& Peebles, P. J. E. 2003, ApJ, 589, 29

Ludlow, A. D., Navarro, J. F., White, S. D. M., Boylan-Kolchin, M., Springel, V., Jenkins, A., \& Frenk, C. S. 2011, MNRAS, 415, 3895

Lynden-Bell, D. 1967, MNRAS, 136, 101

Lynden-Bell, D., \& Wood, R. 1968, MNRAS, 138, 495

Macciò, A. V., Stinson, G., Brook, C. B., Wadsley, J., Couchman, H. M. P., Shen, S., Gibson, B. K., \& Quinn, T. 2012, ApJ, 744L, 9

MacMillan, J. D., Widrow, L. M., \& Henriksen, R. N. 2006, ApJ, 653, 43 
Navarro, J. F., Frenk, C. S., \& White, S. D. M. 1997, ApJ, 490, 493

Navarro, J. F., et al. 2010, MNRAS, 402, 21

Persic, M., Salucci, P., Stel, F. 1996, MNRAS, 281, 27

Pontzen, A., \& Governato, F. 2013, MNRAS, 430, 121

Remus, R.-S., Burkert, A., Dolag, K., \& Johansson, P. H., Naab, T., Oser, L., \& Thomas, J. 2013, ApJ, 766, 71

Rubin, V. C., Ford, W. K. Jr., \& Thonnard, N. 1980, ApJ, 238, 471

Sérsic, J. L. 1968, Atlas de Galaxias Australes (Córdoba: Observatorio Astronómico)

Sharma, S. \& Steinmetz, M. 2006, MNRAS, 373, 1293

Sonnenfeld, A., Treu, T., Gavazzi, R., Suyu, S. H., Marshall, P. J., Auger, M. W., \& Nipoti, C. 2013, ApJ, 777, 98

Taylor, J. E., \& Navarro, J. F. 2001, ApJ, 563, 483

Thomas, J., Jesseit, R., Saglia, R. P., Bender, R., Burkert, A., Corsini, E. M., Gebhardt, K., Magorrian, J., Naab, T., Thomas, D., \& Wegner, G. 2009a, MNRAS, 393, 641

Thomas, J., Saglia, R. P., Bender, R., Thomas, D., Gebhardt, K., Magorrian, J., Corsini, E. M., \& Wegner, G. 2007, MNRAS, 382, 657

Thomas, J., Saglia, R. P., Bender, R., Thomas, D., Gebhardt, K., Magorrian, J., Corsini, E. M., \& Wegner, G. 2009b, ApJ, 691, 770

Tremaine, S., Henon, M., \& Lynden-Bell, D. 1986, MNRAS, 219, 285

Valluri, M., Vass, I. M., Kazantzidis, S., Kravtsov, A. V., \& Bohn, C. L. 2007, ApJ, 658, 731

Vass, I. M., Valluri M., Kravtsov, A. V., \& Kazantzidis, S. 2009, MNRAS, 395, 1225

Vogelsberger, M., Mohayaee, R., \& White, S. D. M. 2011, MNRAS, 414, 3044

Wang, J., \& White, S. D. M. 2009, MNRAS, 396, 709

White, S. D. M., \& Narayan, R. 1987, MNRAS, 229, 103

White, S. D. M., Zaritsky, D. 1992, ApJ, 394, 1

Zemp, M., Gnedin, O. Y., Gnedin, N. Y., \& Kravtsov, A. V. 2012, ApJ, 748, 54 
This preprint was prepared with the AAS IATEX macros v5.2. 

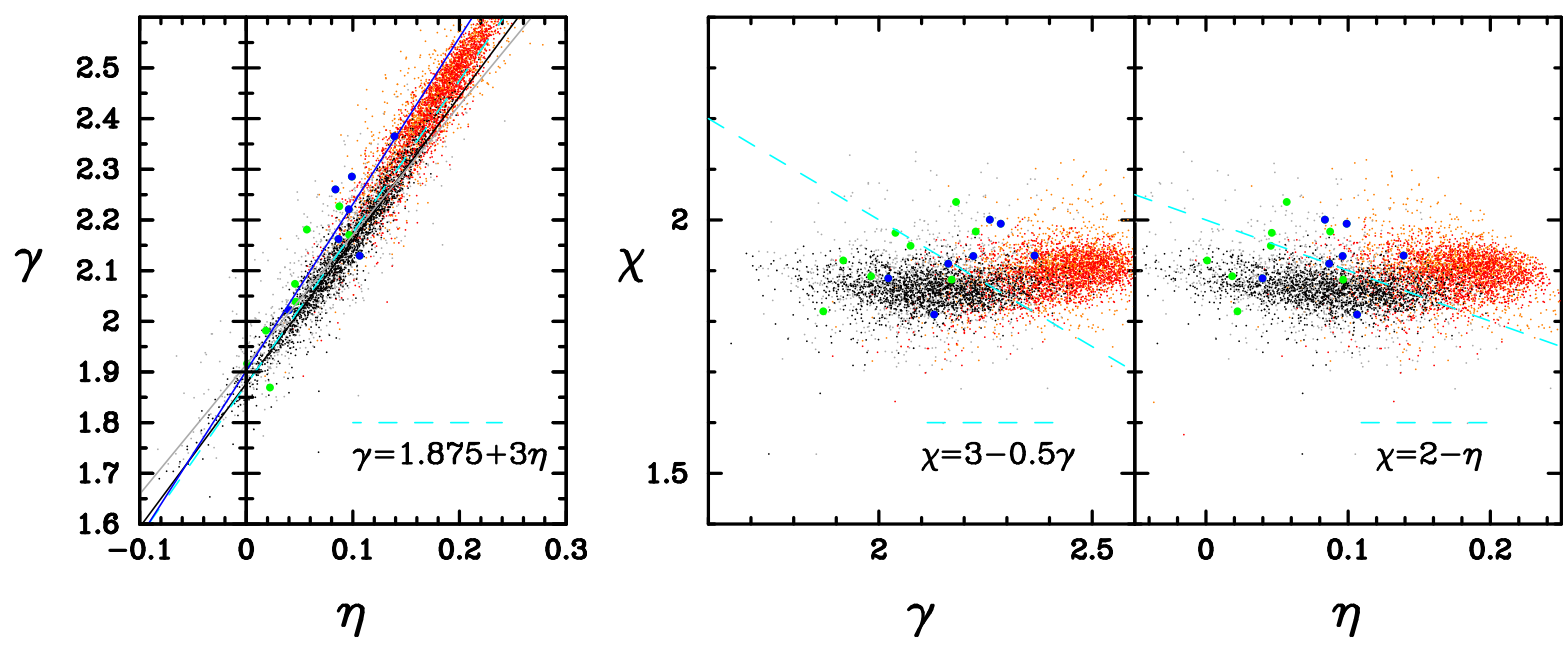

Fig. 1. - Distribution of the slopes $\gamma$ for $\rho(r), \eta$ for $\sigma_{\star \mathrm{r}}(r)$, and $\chi$ for $\rho(r) / \sigma_{\star \mathrm{r}}^{3}(r)$ for $0.1 R_{\mathrm{e}}<$ $r<R_{\mathrm{e}}$ of $\sim 2000$ nearly spherical and rotation-free SDSS galaxies. Black and gray (red and orange) points are respectively for constant and radially varying anisotropies with (without) DM halos. Blue and green points are the values for $0.1 R_{\mathrm{e}} \lesssim r \lesssim 1.5 R_{\mathrm{e}}$, respectively of elliptical and lenticular (S0) galaxies of Coma cluster, with $\sigma_{\star \mathrm{r}}(r)$ replaced by $v_{\star \mathrm{rms}}(r)$. Solid lines are the least-square fit relations. The cyan dashed line $\gamma=1.875+3 \eta$ is the prediction by the classical self-similar spherical infall model (Bertschinger 1985; Fillmore \& Goldreich 1984). The cyan dashed lines $\chi=3-0.5 \gamma$ and $\chi=2-\eta$ are the approximate predictions by Jeans (or virial) equilibrium equation. 

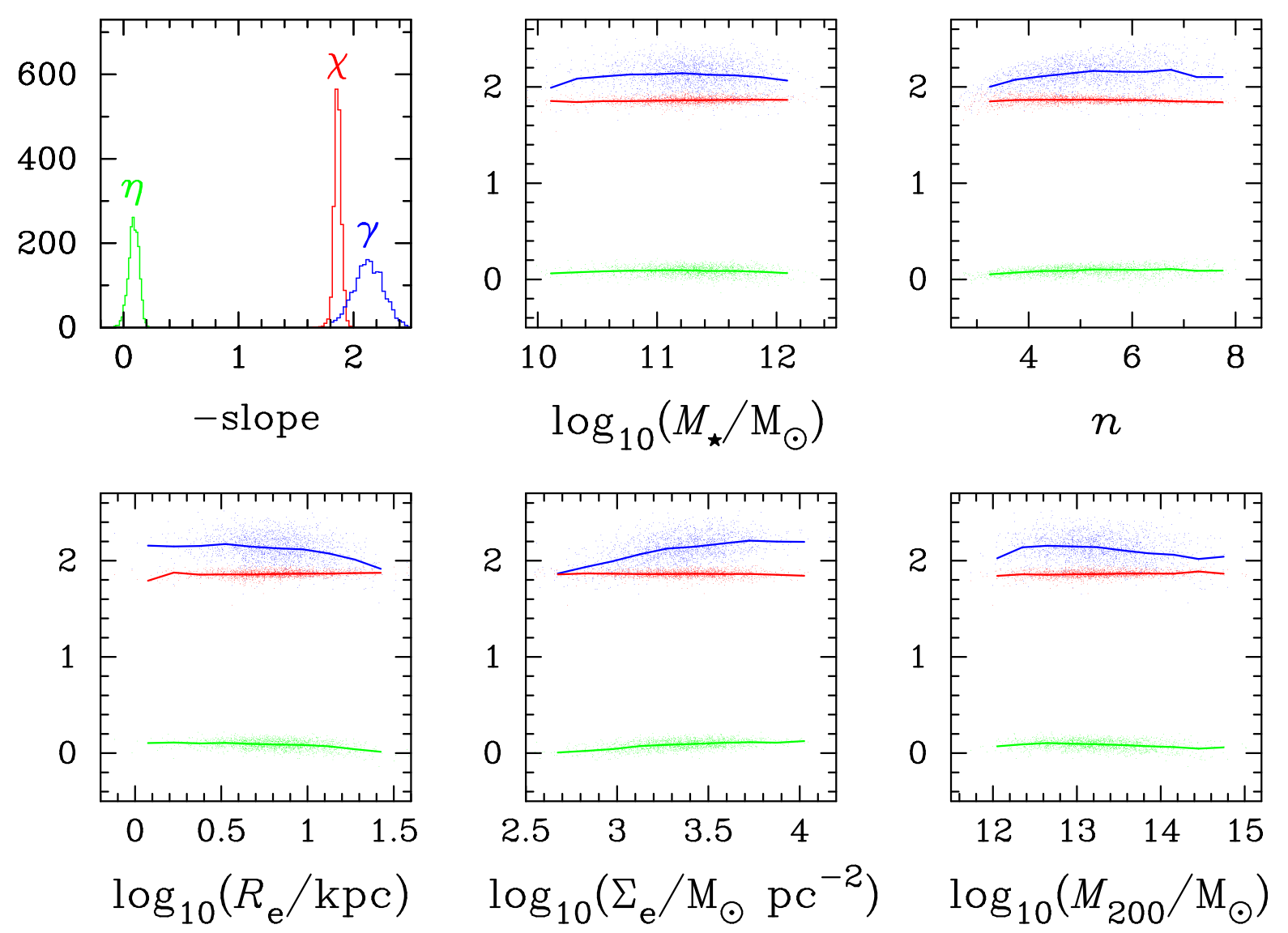

Fig. 2.- Distribution of $\gamma, \eta$, and $\chi$ for the galaxies shown in Figure 1 against various parameters. $\chi$ has the smallest scatter and is not correlated with any parameters while $\gamma$ and $\eta$ show some (anti-)correlations with $R_{\mathrm{e}}$ and $\Sigma_{\mathrm{e}}$. 

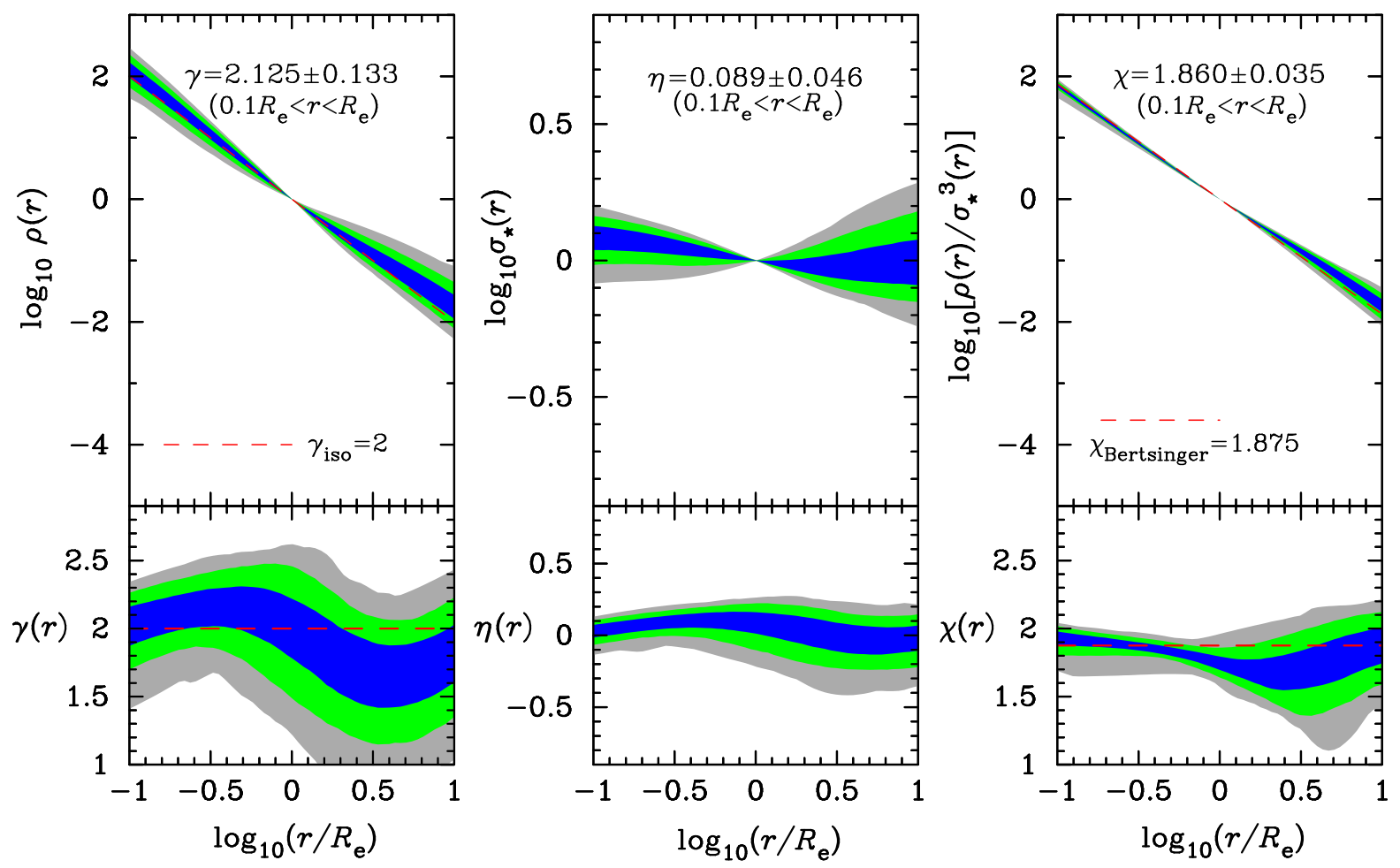

Fig. 3.- Profiles of $\rho(r), \sigma_{\star \mathrm{r}}(r)$ and $\rho(r) / \sigma_{\star \mathrm{r}}^{3}(r)$ for the galaxies shown in Figure 1. All quantities are normalized to the values at $r=R_{\mathrm{e}}$. Blue, green and gray regions contain $68 \%$, $95 \%$ and $99.7 \%$ of galaxies respectively. Bottom panels show profiles of negative slopes. 

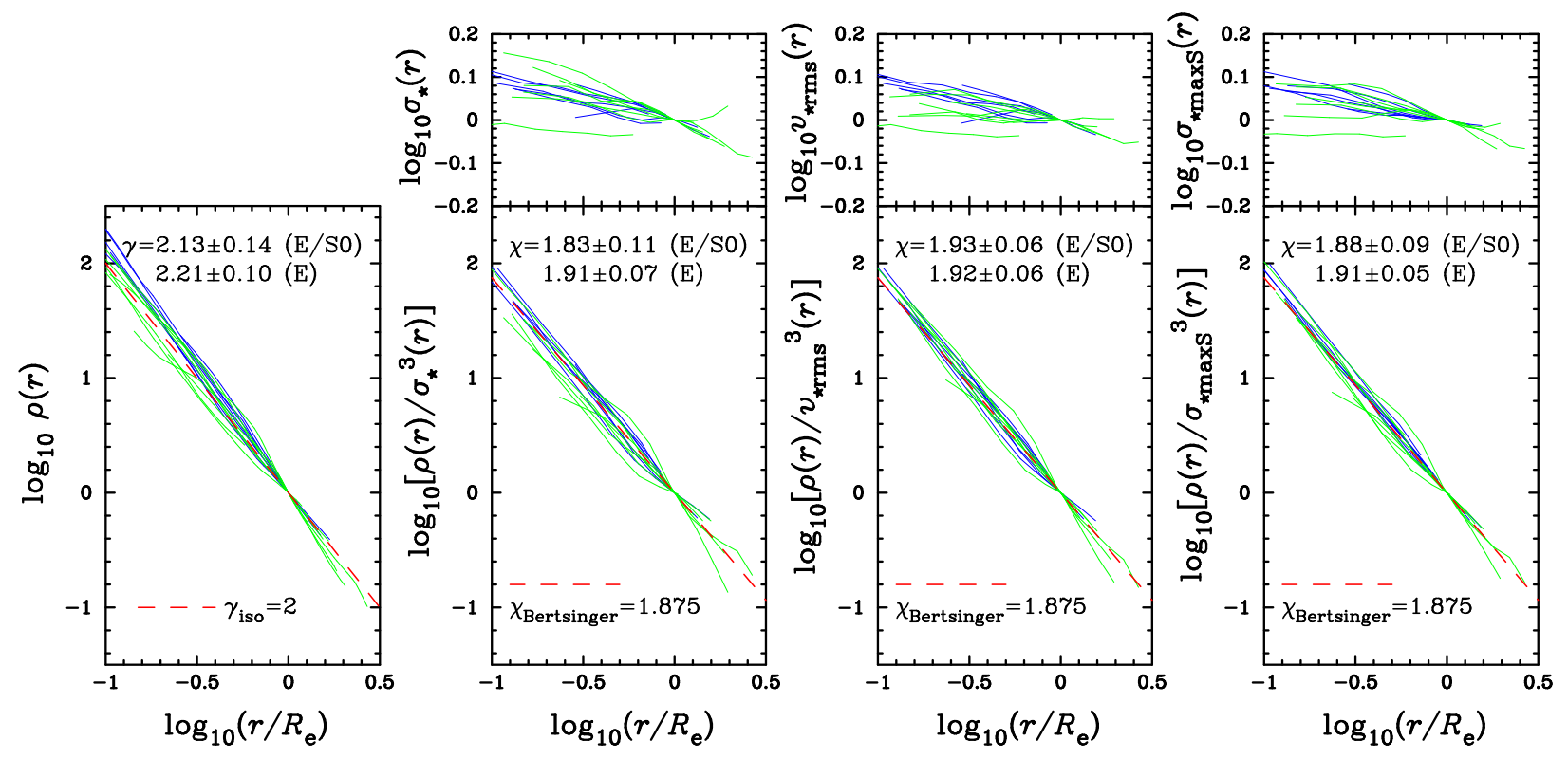

Fig. 4.- Profiles as in Figure 3 but for 15 ETGs of Coma cluster shown in Figure 1 . Note that $v_{\star \mathrm{rms}}(r)$ in general differs from $\sigma_{\star}(r)$ as some ETGs (in particular lenticulars) have non-negligible net rotations. $\sigma_{\star \operatorname{maxS}}(r)$ is the velocity dispersion in models obtained by maximizing entropy but not fitting well observed LOSVDs. 